that is,

$-f d e_{1} d e_{2}\left[\frac{1}{\left\{x_{0}^{2}+\left(1-v_{0}^{2}\right)\left(y^{2}+z^{2}\right)\right\}^{\frac{1}{2}}}-\frac{\left(1-v_{0}^{2}\right) y^{2}}{\left\{x_{0}^{2}+\left(1-v_{0}^{2}\right)\left(y^{2}+z^{2}\right)\right\}^{\frac{3}{2}}}\right]$.

Integrating as in the previous cases, we have

or

$$
-f \cdot \frac{6}{5} \cdot \frac{e^{2}}{2 a}\left\{\frac{1}{v_{0}} \log \frac{1+v_{0}}{1-v_{0}}-\frac{\left(1-v_{0}^{2}\right) \partial}{2 v_{0} \partial v_{0}}\left(\frac{1}{v_{0}} \log \frac{1+v_{0}}{1-v_{0}}\right)\right\}
$$

$$
-f \cdot \frac{6}{5} \cdot \frac{e^{2}}{2 a v_{0}^{2}}\left\{\frac{1+v_{0}^{2}}{2 v_{0}} \log \frac{1+v_{0}}{1-v_{0}}-1\right\},
$$

which gives Abraham's expression for the transverse mass.

CoRNELL UNIVERSITY, March, 1908.

\title{
THE RECENTLY DISCOVERED MANUSCRIPT OF ARCHIMEDES.
}

Professor J. L. Heiberg has published two important accounts of his recent discovery of a new manuscript of Archimedes, both of which are of great interest to mathematicians. The first of these accounts is printed in volume 42 of Hermes. It contains the Greek text of a lost treatise of Archimedes, which is recovered nearly complete in the newly found manuscript. A German translation of the Greek text, and an interesting commentary by Zeuthen, is printed by Heiberg in the Bibliotheca Mathematica, volume 7, page 321.

Professor Heiberg's critical study of Archimedes has extended over a period of more than thirty years. His dissertation, "Quæstiones Archimedeæ" (Copenhagen, 1879), is constantly referred to by students of Archimedes, both on account of its scholarly critique of Archimedes's work, and on account of the innumerable references to Archimedean literature which are there brought together for the first time. In 1880-1881 Heiberg published the definitive edition of Archimedes, with which his name is usually associated. It was while at work upon a second edition of this book that Heiberg's attention was directed to a palimpsest manuscript of mathematical content recently catalogued in a cloister at Constantinople. Failing in an attempt to have the manuscript sent to him at Copen- 
hagen, Heiberg spent the summer of 1906 in Constantinople. Investigation soon showed that the manuscript contained important new material, the study of which would cost more time than was then at Heiberg's disposal. Consequently photography was used wherever possible to copy the pages containing the new material. A facsimile is printed on page 235 of the volume of Hermes referred to above.

The contents of the palimpsest are as follows: (1) Large portions of the previously known writings of Archimedes, the Sphere and Cylinder and On Spirals, nearly complete, parts of Measurement of the Circle, and Equilibrium of Planes ; (2) a large part of the book On Floating Bodies, which was hitherto extant only in a Latin translation, so that numerous defects and lacunæ in this book can now be healed; (3) a hitherto unknown treatise quoted by Heron and referred to by Suidas, the title of which we will translate "A Method." This book constitutes the most important find of new material. There is also

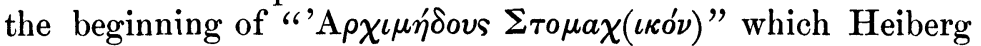
says is a sort of "Chinese game," of which there is not enough remaining to patch up the meaning of the original.

The recovery of this lost work of Archimedes must be considered of the very foremost importance. Of course, the recovery of any work of so original a genius as Archimedes must be considered important. The special significance, however, of the present find consists in the fact that here Archimedes admits us to his mathematical workshop, and points out to us, not only how his discoveries were made, but how other discoveries may be made in the future by the method of research which he discloses. It is a common complaint that the Greek mathematics that has come down to us yields no trace of the process of discovery used by the investigator. The motto of the Greek school might well have been: "Results and not processes are for the public." This book of Archimedes is the one known exception, for herein he relates in charming detail the story of the discovery of some of the most fundamental and most interesting theorems of geometry.

Archimedes sends "A Method" to Eratosthenes, in order to make him acquainted with his method of discovery. It is a conscious attempt, not only to hand Eratosthenes the demonstration of certain theorems which Archimedes had propounded to him, without proof; but to give to Eratosthenes the knowledge of a method of research by which many new truths might 
be expected to be discovered. At this point it is probably best to let Archimedes speak for himself. It is a matter of great interest that the letters which Archimedes prefixed to his different mathematical treatises have come down to us. These are all of very great value in showing us the personal side of his work and in coordinating the different lines of inquiry that absorbed his attention. The present book is no exception, and the letter to Eratosthenes which opens the book is preserved intact. It reads as follows :

"ARCHIMEDES TO ERATOSTHENES, GREETING: I formerly sent you some theorems discovered by myself with the request that you find the proofs which I provisionally withheld. The theorems which I sent to you were as follows :

1. If a cylinder be inscribed in a rectangular prism with square base, so that its bases lie in the opposite squares, and so that elements touch the remaining planes of the prism, and if a plane be passed through the center of the circle which is the base of the cylinder and a side of the square lying in the opposite face of the prism, then this plane cuts off a segment of the cylinder which is bounded by two planes (the cutting plane and the plane wherein lies the base of the cylinder) and by the portion of the cylindrical surface lying between the planes just mentioned; this segment of the cylinder is $\frac{1}{6}$ of the whole prism.

2. If there be inscribed in a cube a cylinder which has its bases in opposite squares and touches the other four faces of the cube, and, moreover in the same cube a second cylinder be inscribed which has its bases in two other squares, and touches the other four faces of the cube, the solid enclosed by the cylindrical surfaces (which solid is common to the two cylinders), is equal to $\frac{2}{3}$ of the entire cube.

"These theorems are essentially different from those communicated by me earlier; the former bodies, the conoids and spheroids and their segments, we compared with the volumes of cones and eylinders, but none of them were found to be equal to a body bounded by planes; on the other hand, each of the present solids, bounded by two planes and cylindrical surfaces, was found equal to solids bounded by planes. I send the proof of these theorems in this book. 
"However, since I see that you are an excellent scholar, and that you are not only a prominent student of philosophy, but also a lover of mathematical research, I have deemed it well to explain to you and put down in this same book a special method whereby the possibility will be offered you to investigate any mathematical question by means of mechanics. And I am convinced that it is quite as useful in the proof of the theorems themselves ; for many of them that were formerly only clear to me through mechanics were afterwards proved by geometry, although the treatment by the former method was not founded on demonstration : indeed, if one has previously gotten a conception of the problem by this method, it is easier to produce the proof than to find it without a provisional conception. Likewise, if one considers the known theorems whose demonstrations were first found by Eudoxus, namely that the cone and the pyramid are respectively one third of the cylinder and prism having the same base and equal altitudes, one must award no slight credit to Democritus who first obtained expression for these truths without a proof. We are also in the position of having previously discovered in the same way the theorems now to be published, and we feel obliged to make the method known, partly because we have previously spoken of them, lest it be believed that we have spread an idle tale, partly in the conviction that there will be instituted thereby a matter of no slight utility in mathematics. In fact, I believe that living or future investigators will discover other theorems which have not occurred to us by the method here discussed.

"We will first explain a theorem that first became clear to me by means of mechanics - to wit that a segment of a parabola is $\frac{4}{3}$ the triangle that has the same base and equal altitude, but thereafter will be given in turn the several theorems found by the method described; and at the conclusion of the book we present the geometrical proof of the theorems above mentioned."

After this introduction, Archimedes gives a list of eight lemmas which he will use in the following demonstrations. All of these are theorems concerning the position of the center of gravity of known geometrical figures. The first five are found in his treatise "On the equilibrium of planes." No. 6, "The center of gravity of a circle is its center"; No. 7, "The center of gravity of a cylinder is the middle point of its axis"; No. 8 , "The center of gravity of a cone divides the axis so that the 
portion adjacent to the apex is thrice the portion adjacent to the base" are not found in any of his existing works. They may be contained in a lost work on centers of gravity, referred to by Simplicius, for Archimedes states that these first eight have already been published. He then states another preliminary theorem, which is the same as "On conoids," 1.

The new method of Archimedes is nearly identical with the method of the integral calculus. Many of the processes used by the ancients had the semblance of the method of the infinitesimal calculus, but the present manuscript shows that Archimedes had taken the decisive step in his method of investigation and had done it consciously. In this work he asserts that surfaces are to be considered as made up of lines, and that solids of revolution are "filled up" by circles. It is true that he expressly admits that his method is not demonstration, but he is thoroughly convinced that the results obtained by it are correct. One must emphasize the fact that the conception and use of the infinite was prohibited in Greek mathematics. The paradoxes of Zeno and the sophists had overfrightened the mathematicians of the Greek school and they had found their only safety in their ingenious theory of proportion and the method of exhaustions. It is an exceedingly interesting fact that Archimedes dared to work with the new idea in his preliminary investigations and it is even more remarkable that he was willing to communicate it to his fellow workers. How very strict the mathematical traditions were on this point is well illustrated by the fact that Archimedes not only gives a geometrical proof for the volume of the segment of a cylinder in the form of the infinitesimal method suggested by his mechanical proof, but he afterwards adds a demonstration in the precise form of a regular exhaustion proof, in order that no one could question its validity.*

Archimedes has abundantly shown that he possessed the genius necessary to formulate the infinitesimal method on a sound basis. He evidently made no attempt of this sort, and it does not appear certain whether his lack of effort in this direction was due to the fact that he saw no means of avoiding in all cases the use of the principles of mechanics in applying the infinitesimal method, and hence the impossibility of a

* These comments are substantially those of Heiberg. For many references given by Heiberg see Hermes, 1. c., p. 302. The last sentence of above paragraph refers to theorems XI, XII, XIII of Archimedes's text. 
demonstration purely geometrical ; or whether he was deterred from such effort on account of adhesion to the traditions of the Greek school. Besides these considerations, there is the strong native desire of great constructive intellects to leave their investigations in the synthetic form - so strongly apparent in the case of Newton and by no means extinct in later days. My own conviction, after some years of familiarity with Archimedes's writings, is that Archimedes was not willing to rest a geometrical demonstration upon mechanical principles, except provisionally, and that his reason was not that rigor could not be secured in that direction, but that there was a scientific propriety, as well as an æsthetic obligation, to keep geometry sufficient unto itself. We all see how such a doctrine fits into the platonic philosophy of Archimedes's time and is in accord with the spirit of the Alexandrian school.

Few realize, except those who have put some study upon Archimedes's work, the tremendously exacting labor that Archimedes put upon himself in placing the demonstrations of his discoveries in the form of exhaustion proofs. To him geometry was the noblest of the sciences. Plutarch may not be exagger-

ating when he says, "he placed his whole delight in these intellectual speculations, which, without any relation to the necessities of life, have an intrinsic excellence arising from truth and demonstration only."

The first two theorems in the new manuscript of Archimedes suffice to illustrate his method. I have abbreviated or omitted descriptive portions of the demonstrations, but have put in his exact language as far as practicable those portions which are of the greatest importance in conveying his exact point of view.

"Theorem I. Let $\alpha \beta \gamma$ be a segment of a parabola bounded by the straight line $\alpha \gamma$ and the parabola $\alpha \beta \gamma$. Let $\alpha \gamma$ be bisected at $\delta$ and let $\delta \beta \epsilon$ be drawn parallel to the diameter of the parabola. Draw $\alpha \beta$ and $\beta \gamma$. Then the segment $\alpha \beta \gamma$ is $\frac{4}{3}$ the triangle $\alpha \beta \gamma$."

"Draw from the points $\alpha, \gamma$ the line $\alpha \zeta$ parallel to $\delta \beta \epsilon$ and the tangent $\gamma \zeta$. Produce $\gamma \beta$ to $\kappa$ and make $\kappa \theta=\gamma \kappa$. We then suppose that $\gamma \theta$ is a 'neck yoke' $*$ with middle point $\kappa$

\footnotetext{
* The Greek $\zeta v \gamma \delta v$ literally means yoke. It is the same word as the Latin jugum or English yoke. It came to be used for beam of a balance and Archimedes uses it as the equivalent of lever, by which it is rendered in translations of Archimedes.
} 
and that $\mu \xi$ is any straight line parallel to $\epsilon \delta$. Since $\gamma \beta \alpha$ is a parabola, $\gamma \zeta$ a tangent and $\gamma \delta$ an ordinate, then $\epsilon \beta=\beta \delta$; in fact this is proved in the Elements. For this reason, and since $\zeta \alpha$ and $\mu \xi$ are parallel to $\epsilon \delta$, we know that $\mu \nu=\nu \xi, \zeta \kappa=\kappa \alpha$. Since $\gamma \alpha: \alpha \xi=\mu \xi: \xi_{0}$ (in fact this was proved in a lemma [Quad. Parab. 5]), we have $\gamma \alpha: \alpha \xi=\gamma \kappa: \kappa \nu$, and since $\gamma \kappa=\kappa \theta$, therefore $\theta \kappa: \kappa \nu=\mu \xi: \xi_{0}$. Now $\nu$ is the center of gravity of the

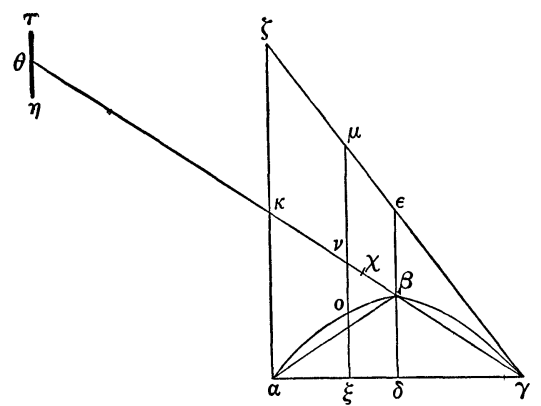

FiG. 1.

straight line $\mu \xi$, since $\mu \nu=\nu \xi$; hence if we construct $\tau \eta=\xi_{0}$ with $\theta$ as its center of gravity, so that $\tau \theta=\theta \eta$, then the straight line $\tau \theta \eta$ must be in equilibrium about $\kappa$ with $\mu \xi$ in the position where it is ; for $\theta \nu$ is divided in the inverse ratio of the weights $\tau \eta$ and $\mu \xi$ and $\theta \kappa: \kappa \nu=\mu \xi: \eta \tau$; therefore $\kappa$ is the center of gravity of the combined weights. In the same way all lines which are drawn in the triangle $\zeta \alpha \gamma$ parallel to $\epsilon \delta$ are in equilibrium, in the position where they are, with their segments intercepted by the parabola, provided these latter be displaced to $\theta$, so that $\kappa$ will be the center of gravity of the combined weights. Since the lines of the triangle $\gamma \zeta \alpha$ make up the triangle, and since the lines corresponding to $\xi_{0}$ make up the segment of the parabola $\alpha \beta \gamma$, therefore the triangle $\zeta \alpha \gamma$, in the place where it is, is in equilibrium about the point $\kappa$, with the segment of the parabola, if this latter be displaced so as to have $\theta$ as center of gravity; hence $\kappa$ is the center of gravity of the combined weights.

"Now let $\gamma \kappa$ be so divided at $\chi$ that $\gamma \kappa=3 \kappa \chi$; then $\chi$ is the center of gravity of the triangle $\alpha \zeta \gamma$; for this is proved in the theory of equilibrium (De plan. æquil, I, 14). Now the triangle $\zeta \alpha \gamma$, in the place where it is, is in equilibrium about $\kappa$ 
with the segment $\beta \alpha \gamma$, if this be displaced so that $\theta$ is its center of gravity, and the center of gravity of the triangle $\zeta \alpha \gamma$ is $\chi$; therefore the triangle $\alpha \zeta \gamma$ : segment $\alpha \beta \gamma$ displaced to $\theta$ as center of gravity :: $\theta \kappa: \kappa \chi$. But $\theta \kappa=3 \kappa \chi$; therefore the triangle $\alpha \zeta \gamma=3$ segments $\alpha \beta \gamma$. But the triangle $\zeta \alpha \gamma=4$ triangles $\alpha \beta \gamma$, since $\zeta \kappa=\kappa \alpha$ and $\alpha \delta=\delta \gamma$; therefore the segment $\alpha \beta \gamma=4 / 3$ the triangle $\alpha \beta \gamma$."

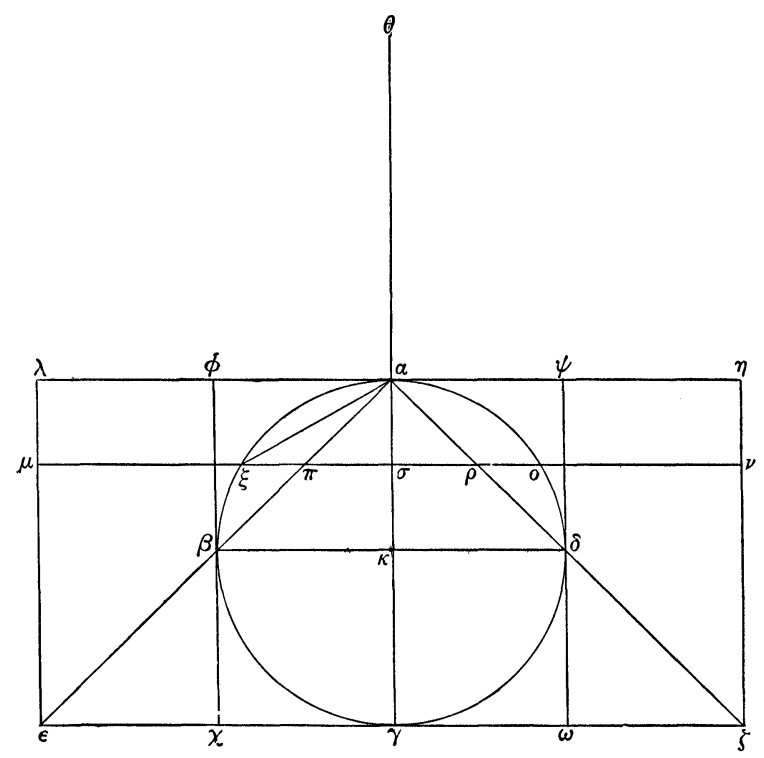

FIG. 2.

"Theorem II. That the sphere is four times as great as a cone whose base is equal to a great circle of the sphere, but whose altitude equals the radius of the sphere; and that a cylinder whose base is equal to the great circle of the sphere, but whose altitude is equal to the diameter of the circle, is one and one half times as great as the sphere, can be explained by the present method in the following manner:

"Let there be (Fig. 2) a sphere and two mutually perpendicular diameters $\alpha \gamma, \beta \delta$ of the great circle $\alpha \beta \gamma \delta$; there is a circle of the sphere perpendicular to $\alpha \beta \gamma \delta$ whose diameter is $\beta \delta$. On this circle let a cone be constructed whose apex is $\alpha$ and afterwards let its convex surface be produced until the conical surface is cut by the plane passing through $\gamma$ parallel to the base 
of the first cone, in consequence a circle is intercepted which is perpendicular to $\alpha \gamma$ and whose diameter is $\epsilon \zeta$. Erect a cylinder on this circle whose axis is $\alpha \gamma$ and having elements $\epsilon \lambda$ and $\zeta \eta$. Produce $\gamma \alpha$ and make $\alpha \theta=\gamma \alpha$, and suppose $\gamma \theta$ to be a lever whose fulcrum is $\alpha$; moreover let any line, as $\mu \nu$, be drawn parallel to $\beta \delta$; it cuts the circle $\alpha \beta \gamma \delta$ in $\xi$ and $o$, the diameter $\alpha \gamma$ in $\sigma$, the line $\alpha \epsilon$ in $\pi$ and $\alpha \zeta$ in $\rho$. Let a plane be passed through the line $\mu \nu$ perpendicular to $\alpha \gamma$; it will cut a circle from the cylinder of diameter $\mu \nu$; it will cut the sphere in a circle of diameter $\xi_{0}$; it will cut the cone $\alpha \epsilon \zeta$ in a circle of diameter $\pi \rho$. Now since $\gamma \alpha \times \alpha \sigma=\mu \sigma \times \sigma \pi$ (for $\alpha \gamma=\sigma \mu, \alpha \sigma=\pi \sigma$ ) and $\gamma \alpha \times \alpha \sigma=\alpha \xi^{2}=\xi \sigma^{2}+\sigma \pi^{2}$, therefore $\mu \sigma \times \sigma \pi=\xi \sigma^{2}+\sigma \pi^{2}$; moreover, since $\gamma \alpha: \alpha \sigma=\mu \sigma: \sigma \pi$ and $\gamma \alpha=\alpha \theta$, therefore $\theta \alpha: \alpha \sigma$ $=\mu \sigma: \sigma \pi=\mu \sigma^{2}: \mu \sigma \times \sigma \pi$. It has been shown, however, that $\xi \sigma^{2}+\sigma \pi^{2}=\mu \sigma \times \sigma \pi$; therefore $\alpha \theta: \alpha \sigma=\mu \sigma^{2}: \xi \sigma^{2}+\sigma \pi^{2}$. But $\mu \sigma^{2}: \xi \sigma^{2}+\sigma \pi^{2}=\mu \nu^{2}: \xi 0^{2}+\pi \rho^{2}=$ the circle of cylinder of diameter $\mu \nu$ : the circle of cone of diameter $\pi \rho+$ the circle of sphere of diameter $\xi_{o}$. Therefore $\theta \alpha: \alpha \sigma=$ the circle of cylinder : circle of cone + circle of sphere. Therefore, the circle of the cylinder, in the place where it is, is in equilibrium about the point $\alpha$ with the combined circles of diameters $\xi_{0}, \pi \rho$, if they be displaced to $\theta$ so that $\theta$ is the center of gravity of both. In the same way it can be shown that if any other line be drawn in the parallelogram $\zeta \lambda$, parallel to $\epsilon \zeta$, and a plane passed through it perpendicular to $\alpha \gamma$, then the circle intercepted by the cylinder is in equilibrium about $\alpha$ with the combined circles intercepted by the sphere and cone, provided they be displaced and so placed on the lever arm at $\theta$ that $\theta$ is the center of gravity of both. If, therefore, the cylinder, sphere and cone be filled up with the above mentioned circles, then the cylinder, in the place where it is, will be in equilibrium about $\alpha$ with the combined cone and sphere, if they be displaced and so placed on the lever arm at $\theta$ that $\theta$ is the center of gravity of both. Now since the solids named are in equilibrium, the cylinder with center of gravity $\kappa$, the cone and sphere displaced as stated with center of gravity $\theta$, then $\theta \alpha: \alpha \kappa=$ cylinder : sphere + cone. But $\theta \alpha=2 \alpha \kappa$ and the cylinder is equivalent to three cones (Euclid, Elements XII, 10); therefore 3 cones $=2$ cones +2 spheres. If two cones be taken away from both members, the cone whose axial triangle is $\alpha \epsilon \zeta$ is equal to two spheres. But the cone whose axial triangle is $\alpha \epsilon \zeta=8$ cones whose axial triangle is $\alpha \beta \delta$, because $\epsilon \zeta=2 \beta \delta$, therefore, the 8 cones named 
are equal to two spheres. Consequently the sphere whose great circle is $\alpha \beta \gamma \delta$ is four times as great as the cone whose apex is $\alpha$ and whose base is the circle of diameter $\beta \delta$ perpendicular to $\alpha \gamma$.

"Through $\beta$ and $\delta$ draw the lines $\phi \beta \chi$ and $\psi \delta \omega$ in the prallelaogram $\lambda \zeta$ parallel to $\alpha \gamma$ and introduce the cylinder whose bases are circles having the diameters $\phi \psi$ and $\chi \omega$, and whose axis is $\alpha \gamma$. Then the cylinder whose axial parallelogram is $\phi \omega$ is twice as large as the cylinder whose axial parallelogram is $\phi \delta$, and this last is threefold as large as the cone whose axial triangle is $\alpha \beta \delta$, as is proved in the Elements. Therefore the cylinder whose axial parallelogram is $\phi \omega$ is sixfold as large as the cone whose axial triangle is $\alpha \beta \delta$. It has been proved, however, that the sphere of great circle $\alpha \beta \gamma \delta$ is fourfold as large as the same cone; consequently the cylinder is $\frac{3}{2}$ the sphere, which was to be proved.

"Because of this theorem - that a sphere is fourfold as large as the cone whose base is a great circle and whose altitude is the radius of the sphere - the thought occurred to me that the surface of the sphere is fourfold as great as its great circle, because I proceeded from the conception that as a circle is equivalent to a triangle whose base is equal to the circumference of the circle and whose altitude is equal to the radius of the circle, in the same way a sphere is equal to a cone whose base is equal to the surface of the sphere, and whose altitude is equal to the radius of the sphere."

Following the above theorems, Archimedes gives a number of others, all of which are investigated by the same method. Among these are : volume of a spheroid; volume of a paraboloid ; center of gravity of a paraboloid ; center of gravity of a hemisphere; volume of spherical segment; center of gravity of a spherical segment. Then follows the theorem concerning the volume of the cylindrical segment announced in the introduction. This contains a number of lacunæ. No trace is left of the theorem concerning the solid common to two intersecting cylinders, and of the geometrical proof of the area of parabolic segment, etc., which were promised in the introduction.

The two theorems reproduced above illustrate the method used by Archimedes in the remaining theorems. The method may be summarized as follows: The elements of a solid or surface to be measured are connected by a proportion with the elements of bodies previously considered, and in such manner that 
two terms of the proportion shall be straight lines. The straight lines may then be used as "lever arms" to balance the elements or certain combination of elements selected from the new and old bodies. Then if the magnitude and position of the centers of gravity of the old bodies be known, the new body, when displaced so that its center of gravity lies at one end of the lever arm, will be in equilibrium with a known magnitude "in the place where it is" and hence an equality is established. This means, in modern language, that a definite integral is evaluated by means of the mean value of a function defined by means of the center of gravity. On the other hand, if the magnitudes of the bodies under investigation be known, the center of gravity of one of them can be found by the same process. The method requires the introduction of the principle of moments as a postulate of mathematics and a formulation of the doctrine of infinitesimals. Archimedes avoided this by the construction of exhaustion proofs.

A number of matters of considerable historical interest are settled by the present work. It is now known that Democritus and not Eudoxus is to be given credit for the discovery of the relation between the volume of the pyramid and prism and of the cone and cylinder. Evidently Eudoxus was the first to put the demonstrations in the form of an exhaustion proof, but his work is to be compared with Archimedes's geometrical demonstrations constructed after the facts had been developed by means of mechanics. It is also of interest to note that Archimedes states that he discovered the theorem concerning the volume of the sphere previous to the discovery of the theorem expressing the area. His description of the manner in which the idea occurred to him that the surface of the sphere is fourfold its great circle, is of the very greatest interest. One would naturally but erroneously infer from the way the matter is put in his treatise on the Sphere and Cylinder, that the area of the sphere became known to him before the discovery of its volume.

Archimedes makes it clear that he is using this method to "discover" the theorems, and that it is " not founded on demonstration." But in speaking of spheres as "filled up" by circles and of surfaces as "made up" of lines, he is not misusing the method of infinitesimals nor treading on dangerous ground. In fact the elements which "fill up" the magnitudes are always so taken by Archimedes that the process can be immediately satisfied by an exhaustion proof. From this point of view his 
scheme may be regarded as analogous to the modern method of infinitesimals when founded upon the doctrine of limits. There is a normal and systematic procedure, although tedious and laborious, for converting the mechanical proofs into exhaustion proofs. Therefore Archimedes may be regarded as having taken the decisive step in founding a method which in essential respects is that of the integral calculus. If he had been like many modern mathematicians, he would have omitted the exhaustion proofs altogether, but would have added to each of his mechanical proofs a set phrase like this : "It is easy to see that an exhaustion proof may be constructed in the usual manner. This is left as an exercise for the reader."

Charles S. Slichter.

\section{SHORTER NOTICES.}

Mehrdimensionale Geometrie, II Teil, Die Polytope. Von Professor Dr. P. H. Schoute. Leipzig, G. J. Göschen (Sammlung Schubert XXXVI). 1905. ix + 326 pp.

THE second (and final) volume of this work, like the first, is worthy to be associated with the other excellent books of the Schubert collection. Comparatively little of the subject matter is new, but a large number of interesting and useful results have been gathered together in a convenient form. The entire volume is devoted to the treatment of the polytop, which the author defines, for space of $n$ dimensions, as any portion of that space enclosed in any manner whatever. The first 262 pages treat the linear polytop, $i$. e., one bounded by flat spreads of $n-1$ dimensions $\left(R_{n-1}\right.$ 's); while the remaining 64 pages are concerned with the hypersphere, cone, cylinder, and rotation spread.

Under the heading "Topologische Einleitung," the first section treats (among other things) the simplex, and its various sections and projections ; the question of a general classification of polytops; the definition of hyper-pyramids and prisms and the $n$-dimensional analogues of other special polyhedra, such as the truncated pyramid and prism, the frustums, etc.; and finally discusses the Euler law and its $n$-dimensional extension. In this treatment of the Euler law, thirteen pages are devoted to the well-known three-dimensional case, four different methods 